\title{
Smart garments for performance and training assessment in sport
}

\author{
Giuseppe Andreoni, Paolo Perego, Marcello C. \\ Fusca, Riccardo Lavezzari \\ Design Dept. Politecnico di Milano \\ Milan, Italy \\ \{giuseppe.andreoni, paolo.perego, marcello.fusca\}@po \\ limi.it
}

\author{
Giorgio C. Santambrogio \\ DEIB Dept. Politecnico di Milano \\ Milan, Italy \\ giorgio.santambrogio@polimi.it
}

\begin{abstract}
This paper describes a novel method and system to monitor and compute swimmer performance. We placed a 3-axes accelerometer onto the athlete's back. This accelerometer allows for detecting and interpret single gestures during the training session. The acquired data can be processed to compute synthetic parameters for a qualitative assessment of the performance. Symmetry and no. of foot-/arm-stroke integrate the measurement. 3 healthy volunteers (1 male, 2 females) participated to the system validation. The system proved a fine detection of each phase and movement in the analyzed sport. Also comfort and wearability was positively assessed.
\end{abstract}

Keywords-component; wearable monitoring; sport; swimming; style detection; performance assessment.

\section{INTRODUCTION}

Wearable Technologies are garments and accessories embedding advanced sensors and systems capable to measure, process and transmit data from the users' body. Many applications have been explored and its exploitation is now really starting [1]. Medical and Sport are the main ones. In medicine, sensorized garments allow for long term monitoring of patients through the so-called non-intrusive paradigm; a process almost transparent to the subject because sensors are integrated into the natural interface of our body towards the external environment, i.e. clothes. Monitoring devices have been also miniaturized and optimized in power consumption and performances greatly improving non-intrusiveness. Sport is another application in rapid expansion. Athletes' performance can be monitored during exercise. A new challenge is constituted by water sports were these devices can be critically used to collect the useful data.

Swimming is a cyclic sport whose goal is the speedy and continuous water propulsion. The performance of swimming is strongly related to the swimmer's technique. Therefore, a swimmer who wants to improve his swim performance has to devote a substantial proportion of the training to his swim style improvement. Apart from a swimmer's effort of heading forward efficiently, the swimmer needs to be concerned about breathing and floating at the water surface. The two most important physical principles to swim faster and more efficiently are a) the reduction of water resistance improving body balance and body rotation, b) the increase of the propelling force by improving the arm strokes and hence the stroke efficiency. The consensus nowadays is that both factors must be optimized [2].
Technique and coordination are strategic and minimal changes could results in significant performance modification.

Nowadays the use of technology in sports performance analysis is a rapidly increasing practice. Tools for analysis aim to provide useful information to improve coach knowledges and athletes feedback. In recent years, video based analysis of swimming performance is the most used method and allows the calculation of stroke rate, stroke length and assessment of the general characteristics of a swimmer's style; for example, angles of arm joints or degrees of body roll. Video analysis uses either two or three cameras placed at various positions in and/or above the pool.

An increasingly widely adopted alternative to video-based analysis employs small electronic accelerometers located at various sites on the swimmers body. The use of accelerometers in athletic performance monitoring has been validated by numerous studies covering a range of disciplines including: ambulatory measurements [3], physical activity [4], gait analysis [5], orientation and movement [6] and to improve athlete performance [7].

This work describes a new system for monitoring a swimmer through a single motion sensor and sensorized swimwear to detect Heart Rate (HR).

\section{MATERIALS AND METHOD}

\section{A. Monitoring device}

We developed a miniaturized system measuring 1 ECG lead and 3-axis accelerations; data are stored onboard and can be downloaded via Bluetooth module. The wearable actigraph is based on the 3-axis accelerations sensor (LIS3L06AL, STMicroelectronics), a Bluetooth ${ }^{\odot}$ transmission module (PAN1540, Panasonic), a STM32 microcontroller and a rechargeable Li-Ion battery, all packed in a plastic waterproof case of $5 \times 58 \times 25 \mathrm{~mm}$. The choice of the Bluetooth technology for data transmission at this stage was due to the very large diffusion of this technology in any device for data computing or visualization such as PC or tablet or smartphone. Obviously real time data display was not developed because of the water immersion of the device which makes wireless transmission impossible. We explored two methods for solving this issue: 1) the adoption of an antenna emerging from water at trunk or head level, but preliminary user tests evidenced unacceptance of this solution by the swimmers even in a prototypal stage for its critical wearability; 2) the use of a 
specific transceiver for water applications in data transmission. This second option is the real possibility to be developed in the near future, but at this stage of technological proof of concept we decided to set up the device as data logger and off-line data analysis.

\section{B. Preliminary setup assessment}

After a preliminary analysis of the size and shapes of the male and female swimsuit and a related interview with 2 professional swimmers (1 male and 1 female) and their coaches, a wearability study correlated with data quality was conducted with 3 amateur swimmers. Five positions of the unit were analyzed: 1) centrally on the front of the chest, 2) on the back of the trunk in correspondence of the 9th thoracic vertebra, 3) back on the pelvis in correspondence of the 5th lumbar vertebra, 4) frontally on the pelvis in correspondence of the right superior anterior iliac spine, 5) back on the neck in correspondence of the 7th cervical vertebra (please refers Fig. 6 for precise positions).

The goal was to use a single device to gather information about styles, global propulsion performance, arm-stroke count, foot-stroke count, symmetry of the movement.

\section{RESULTS AND DISCUSSION}

In the following figures the graphs of the 3D Accelerations measured during swimming in the three different styles are shown.

The graphs of the 3-axis accelerations clearly interpret the swimming gestures. Thus a first discussion could be done about the relation between quality of data and anatomicallandmark where the recording unit is placed.

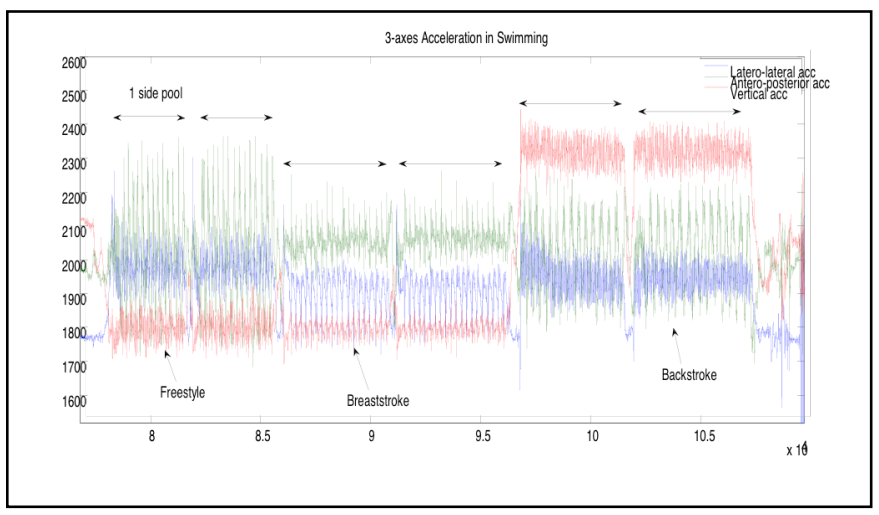

Figure 1. Example of a 3-style swimming test. On the horizontal axis is time in samples, on the vertical axis is acceleration in samples $( \pm 2 \mathrm{~g}$ over a 3600 sampling scale). In blue the latero-lateral accelerations, in red the vertical acceleration, and in green the antero-posterior accelerations are shown.

The selected position onto the body (i.e. on the back in correspondence of the 9th thoracic vertebra) answers to the given requirement of having just a single recording unit: all the styles are evidenced and they demonstrate a peculiar pattern. This allows for an automatic style classification by a dedicated algorithm. For instance the acceleration along the vertical axis (the red line in all the figures) is reversed with respect to the midline in the case of the backstroke, while the classification between freestyle and breaststroke is easy implemented through a pattern classification on the antero-posterior accelerations (in green).

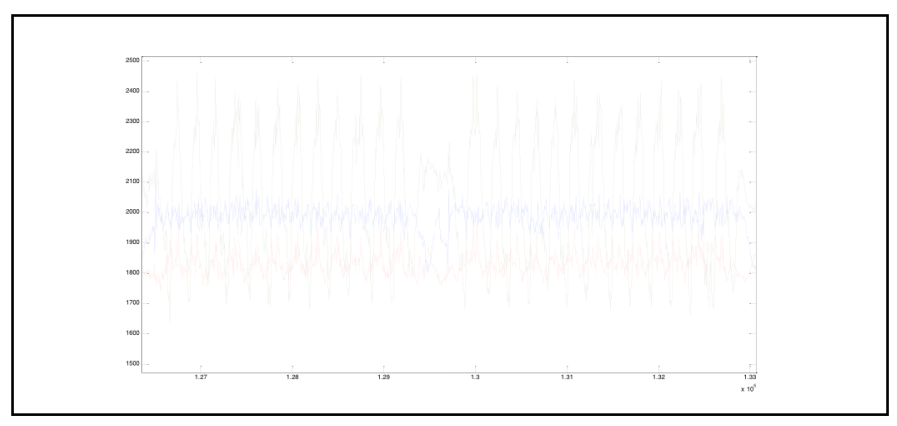

Figure 2. 3-axis accelerations recorded during freestyle monitoring

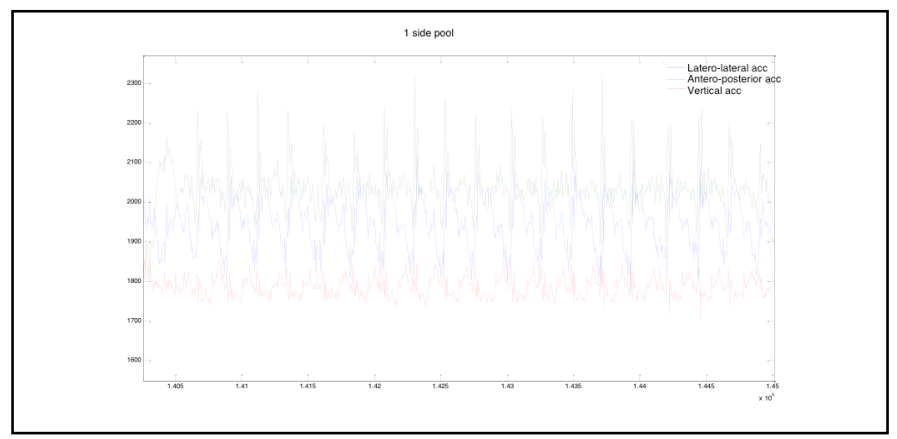

Figure 3. 3-axis accelerations recorded during breast-stroke monitoring.

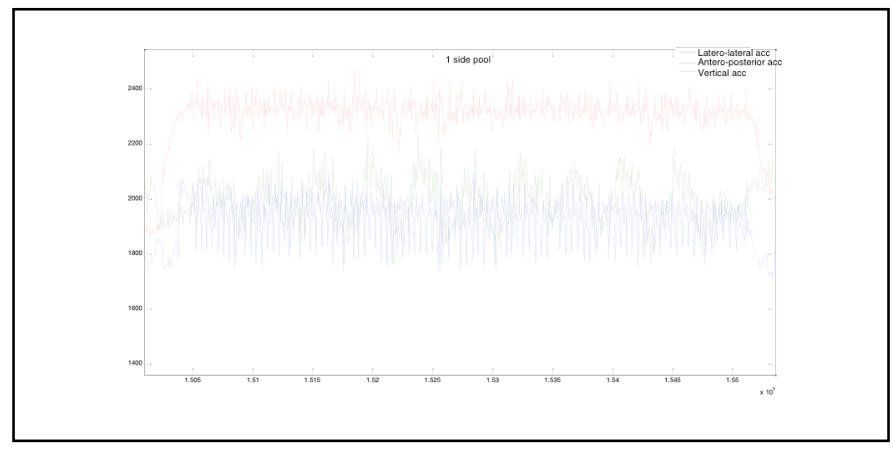

Figure 4. 3-axis accelerations recorded during backstroke monitoring.

The changes in directions are also clearly identified both in time and the propulsion force at the start, thus determining the time for completing each single pool. This is an important parameter to be considered during training of top athletes.

If the graph presenting the single style pattern are analyzed, it is clearly visible how it is easy to identify the number of armstrokes, their efficiency in propulsion (a rough estimate is given by the amplitude of each motion) and their symmetry, computed as the difference in amplitude between two consecutive peaks, taking as zero level the median line.

A similar approach is used for backstroke, in which armstroke frequency is lower. For breaststroke style a double peak is shown for every propulsive act (Figure 3): there is a small difference in time between the arm-stroke and the leg-stroke. This parameter is interesting for a best synchronization of the body motion during the event. The high peaks variability is due to the amateur swimmer recruited in the test. Another interesting outcome is the possibility to elicit and compute the number of foot-stroke, in particular during backstroke when leg contribution to the total propulsion is greater than in the crawl. The observed effect is particularly evident in the latero-lateral acceleration (in blue in Fig. 4). 


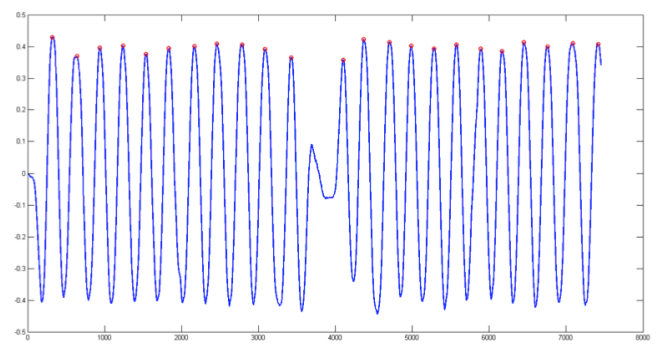

Figure 5. Example of the automatic arm-stroke computation in freestile. The blue line represents the filered accelerations along the antero-posterior axis, and a simple peak detection algorithm identify the peaks (circled in red).

Each single peak corresponds to a footstroke and it is also possible to discriminate between left and right event. If so, by observing the correspondent antero-posterior acceleration it is possible to verify an asymmetric motor pattern to be corrected by intensive training.

A similar approach was followed while defining the best position of the HR electrodes to be integrated into the swimwear.

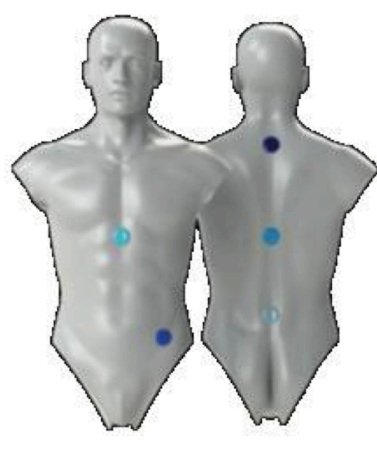

Accelerometer positions

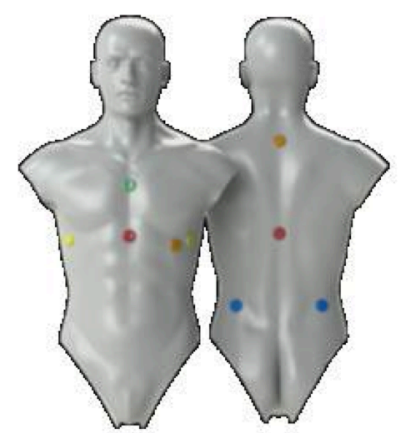

HR Electrodes positions
Figure 6. Accelerometer positions and electrodes positions tested to determine the best recording setup in swimming monitoring.

A parallel analysis demonstrated that the best electrodes positions for HR detection is frontally on the lateral side of chest wall in correspondence of the 10th rib. The experimental setup consisted in three steps:

- A preliminary study with standard $\mathrm{Ag}-\mathrm{AgCl}$ electrodes mounted under a swimsuit and recording signal in a dry environment with the 3 swimmers lying on a small wooden flat plane supporting the back but leaving the possibility to move the limbs: in this way they can simulate the swimming movements.

- Data analysis for selection of the best electrode setup.

- Realization of a sensorized belt with textile electrodes worn under the swimsuit. This setup was tested both in dry environment and also 1 trial in the swimming pool.
This setup produced the minimum motion artefacts and the possibility to have an R-R peak detection for the computation of the actual beat-to-beat heart rate value. As shown in figure 7 the best situation is while swimming with breaststroke style; in this case there is a very stable signal in which the QRS complex is well visible and detectable with standard algorithms. Instead, in freestyle and backstroke there is a baseline fluctuation. This happens for a joint combination of two concurring events:

- The skin-electrodes relative movements, also named motion artefacts: even if the sensorized belt is embedded in the swimwear which is usually very tight to the swimmer, a certain movement of the electrode over the skin is always present. This is also due to the changes in muscles' volume that are intrinsic in the gesture.

- The contribution of the electrical activity of the muscles that produce non symmetrical high frequency signal. This makes the baseline to fluctuate around a central level. Being breaststroke style symmetrical for the upper limbs, this effect is reduced and therefore the signal in this style is better than in other two ones where the movements of the upper limbs are opposite or better in counter-phase.

The possibility to have a joint ECG and motion detection is novel and opens new possibilities in swimming training. The ECG monitoring can be useful to verify the training level and the fatigue of the athletes. This allow for preparing personalized workouts and exercises. A similar approach could also be proposed in medicine to study with specificity the different application situations to design and fit the best sensors setup.

A set of optimized algorithm is under development to carry out a real time analysis to provide immediate feedback to the coach and the swimmer during training. The technical development we are facing is data transmission in/out water for real-time monitoring. In parallel we are developing a new swimsuit with an integrated pocket to insert the electronic unit, and two textile electrodes for ECG monitoring. The under development swimsuit will preserve water resistance both in situ (i.e. where ECG electrodes are) and over the entire electric path for ECG signal. A preliminary validation of the algorithm for automatic style detection is in progress with a video based technique comparison, but it has not been reported in this paper.

\section{CONCLUSION}

A novel system for swimming analysis based on wearable technology is described in this paper. The same system could be easily ported into other sports but, above all, we designed and subsequently demonstrated that our methodological approach can be a very useful pathway also for medical applications: we are conducting a similar work in AAL (Ambient Assisted Living) for elderly. Future development will concern the real-time data transmission (as discussed in the previous section) and a rigorous validation and the extension of the sports, e.g. in soccer and sky race. Preliminary findings demonstrated good results. On this point we already explored 


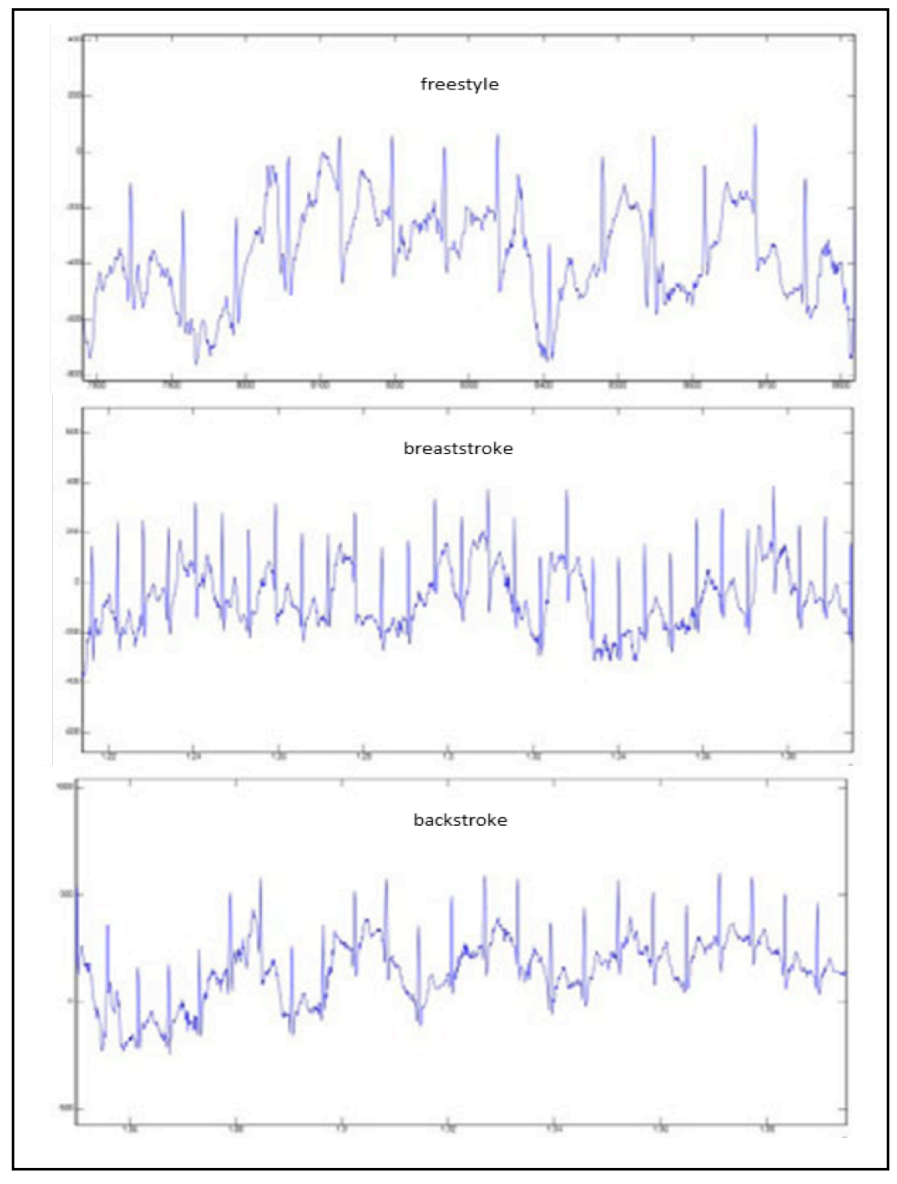

Figure 7. An example of the 1 lead ECG recordings in the three different styles. the possibility to build a sensors network for multiplayers sports both in water and others.

For long range monitoring in open spaces we adopted a ZigBee transceiver collecting data and hopping (to better cover the playfield) over 3 players to the receiving station. In this case we will speak of team performance monitoring.

\section{REFERENCES}

[1] G. Andreoni, M. Bernabei, P. Perego, A. Barichello, L. Piccini, Example of Clinical Applications of Wearable Monitoring System, International Journal of Computer Research. Nova Science Publishers inc. 2011, Vol. 18, No. 3/4, pp 323-39.

[2] M. Bächlin, G. Tröster, Swimming performance and technique evaluation with wearable acceleration sensors, Pervasive and Mobile Computing vol. 8, 2012, pp. 68-81.

[3] J.B.J. Bussmann, W.L.J. Martens, J.H.M. Tulen, F.C. Schasfroort, H.J.G. van den Berg-Emons and H.J. Stam, Measuring Daily Behaviour Using Ambulatory Accelerometry: The Activity Monitor, Behaviour Research Methods, Instruments and Computers, 2001, vol. 33, no.3, pp. 349-356.

[4] Bao, L. and Intille, S.S., Activity Recognition from User-Annotated Acceleration Data", in: A. Ferscha and F. Mattern, eds., Lecture Notes in Computer Science, Springer-Verlag, Berlin, Germany, 2004, pp. 1-17.

[5] J.A. Levine, P.A. Baukol and K.R. Westerterp, Validation of the Tracmor Triaxial Accelerometer System for Walking, Medicine and Science in Sports and Exercise, 2001, vol. 33, no. 9, pp. 1593-1597.

[6] D. Roetenberg, P. Slycke, A. Ventevogel and P.H. Veltink, A Portable Magnetic Position and Orientation Tracker, Sensors \& Actuators, A: Physical, 15 April 2007, vol. 135, no. 2, pp. 426-432.

[7] Anderson, R., Harrison, A.J. and Lyons, G.M., “Accelerometer Based Kinematic Biofeedback to Improve Athletic Performance", in: Ujihashi, S. and Haake, S., eds., Engineering in Sport 4, 2002, 803-809.I. S. Jacobs and C. P. Bean, "Fine particles, thin films and exchange anisotropy, in Magnetism, vol. III, G. T. Rado and H. Suhl, Eds. New York: Academic, 1963, pp. 271-350. 